\title{
Chironomid (Diptera, Chironomidae) species assemblages in northeastern Algerian hydrosystems
}

\author{
N. Chaib, ${ }^{1,2}$ A. Fouzari, ${ }^{2}$ Z. Bouhala, ${ }^{2}$ B. Samraoui, 2,3 B. Rossaro4 \\ 1Département de Génie des Procédés, Faculté de Technologie, Université du 20 Août 1955, \\ Skikda, Algeria; ${ }^{2}$ Laboratoire de Recherche et de Conservation des Zones Humides, Université du \\ 8 Mai 1945 Guelma, Algeria; ${ }^{3}$ Center of Excellence for Research in Biodiversity, King Saud \\ University, Riyadh, Saudi Arabia; ${ }^{4}$ Department of Food, Environmental and Nutritional Sciences \\ (DeFENS), Università degli Studi di Milano, Milano, Italy
}

\begin{abstract}
The aim of this paper was to analyze the distribution of chironomids (Diptera, Chironomidae), and determine their substrate preferences, from two hydrosystems located in northeastern Algeria: the Kebir-East and the Seybouse wadis. Sixty-five species were recorded in 49 sampling sites distributed along the main courses of the two hydrographic nets and their tributaries. The majority of taxa comprised cosmopolitan species widely distributed along these two hydrosystems. Cricotopus (Cricotopus) bicinctus showed the highest abundance and frequency of occurrence (29.52\%) and was widespread in almost all the sampling sites. Species richness ranged from 4 to 23 , Shannon diversity between 0.15 and 0.90 , Evenness from 0.23 to 1 . A cluster analysis was carried out to represent the different groups of sites sharing similar species composition. Agglomerative cluster analysis grouped the sampling sites into four clusters according to the community data. An Indval analysis was
\end{abstract}

Correspondence: Nadjla Chaib, Département de Génie des procédés, Faculté de Technologie, Université du 20 Août 1955, BP 26 Route El-Hadaëk, Skikda, Algeria.

E-mail: nadjla21@yahoo.fr

Key words: Algeria, Chironomidae, spatial distribution, substrate-type, Indval analysis, cluster analysis.

Acknowledgements: the work was supported by the Algerian Ministère de l'Enseignement Supérieur et de la Recherche Scientifique (DGRSDT/M.E.S.R.S.) and the King Saud University Deanship of Scientific Research, Research Group Project No: RGP-VPP-135. DSFP, King Saud University, Saudi Arabia.

Received for publication: 11 October 2012.

Revision received: 12 November 2012.

Accepted for publication: 21 December 2012.

CCopyright N. Chaib et al., 2013

Licensee PAGEPress, Italy

Journal of Entomological and Acarological Research 2013; 45:e2 doi:10.4081/jear.2013.e2

This article is distributed under the terms of the Creative Commons Attribution Noncommercial License (by-nc 3.0) which permits any noncommercial use, distribution, and reproduction in any medium, provided the original author(s) and source are credited. then carried out to detect indicator species for each group of the sampling sites. Cricotopus (Isocladius) sylvestris was indicator of the first group of the sampling sites. Orthocladius pedestris, Rheocricotopus chalybeatus and $C$. bicinctus were indicators of the second group, and Polypedilum cultellatum of the third group. The fourth group was not characterized by any species. Indval analysis allowed also to determine species preferences for substrate size: Corynoneura scutellata and Dicrotendipes nervosus emphasized a preference to fine gravel, and Glyptotendipes pallens to fine sand.

\section{Introduction}

Mediterranean wetlands are under tremendous pressure due to numerous factors like demography, human encroachment and climate change (Hollis, 1992; Hulme et al., 2001). Loss of wetland biodiversity can only be mitigated through critical knowledge of threats (Battisti $e t$ al., 2008; Gibbs, 2000). Such knowledge is compromised when local biodiversity is not well understood, as is the case of northeastern Algeria which houses a wide spectrum of wetlands, many of international importance (Samraoui \& Samraoui, 2008). Despite their ecological and biogeographical interests, the aquatic communities of northeastern Algeria have attracted few systematic studies (Samraoui \& Menai, 1999; Samraoui \& Corbet, 2000; Annani et al., 2012; Samraoui et al., 2012).

The Chironomidae (Diptera) constitute a highly diversified group of aquatic insects frequently occurring in high density in different kinds of ecosystems (Coffman \& Ferrington, 1984). The Chironomidae are of great significance in the structure and function of lotic systems due to their great abundance, diversity and occurrence (Cranston, 1995). The larvae of this family are fundamental components in freshwater food webs, occupying different habitats within river basins, with their distribution determined by several factors; among them, substrate size has an important role in the spatial distribution of macroinvertebrate assemblages (Sanseverino \& Nessimian, 2001; Brooks et al., 2005).

The understanding of the relationship between species and environment is essential; therefore, every assessment will be more accurate if habitat preferences and indicator species are known (Legendre \& Legendre, 1998; McGeoch \& Chown, 1998; Tickner et al., 2000). Despite their importance, little is known of habitat preferences of chironomids, especially in the southern Mediterranean, including Algeria (Lounaci et al., 2000a; Lounaci et al., 2000b; Arab et al., 2004; Belaidi et al., 2004; Chaib et al., 2011a; Chaib et al., 2011b).

Sampling in several wadis (water courses with very irregular hydro- 
logic regime) in Kabily du Djurdjura, northern Algeria (Moubayed et al., 2007) generated a list of 87 chironomid species from this area: 8 belonged to Tanypodinae, 3 to Diamestinae, 57 to Orthocladinae and 19 to Chironomiae; 10 species were not described. A total of 53 species were new records for Algeria, 25 of which being also new records for North Africa.

A survey of Chironomids from the Kebir-East wadi and its tributaries in northeastern Algeria (Chaib et al., 2011b) generated a list of 37 widespread chironomid species in the Palearctic. They include 5 Tanypodinae, 15 Orthocladiinae, 4 Tanytarsini and 13 Chironomini.

A similar study was carried out in the Seybouse basin, where 45 chironomid species were collected.

This study aims to analyze the composition of Chironomidae assemblages in the northeastern Algerian hydrosystems along the Kebir-East and the Seybouse wadis. The spatial distribution of the assemblages was examined and chironomids were correlated with substrate size in order to investigate substrate preferences.

\section{Materials and methods}

\section{Study area}

Forty-nine sampling sites were chosen along the main course of the Kebir-East (23) and the Seybouse wadis (26) and their tributaries on the base of land-use and anthropogenic impacts (Chaib \& Samraoui, 2011; Chaib et al., 2011a, 2011b; Khelifa et al., 2011) (Figure 1, Table 1).

According to the subdivision of hydrographic nets in the eastern region of Algeria by the Agence des Bassins Hydrographiques (ABH$\mathrm{CSM}$ ), our two river systems, the subject of this paper, belong to two different basins: i) the Kebir-East belongs to the watershed of the Côtiers Constantinois Est in the extreme north-east of Algeria, and covers a catchment area of $1600 \mathrm{~km}^{2}$; and ii) the Seybouse basin is the largest sub-basin in the northeastern region, covering an area of $6570 \mathrm{~km}^{2}$.
These two fluvial systems are an important source of water in the northeastern Algeria, since they supply water for irrigation of large agricultural areas extending from the regions of Guelma to El Kala.

Both the Seybouse and Kebir-East basins represent a mosaic of geomorphodynamic natural conditions, as well as diverse levels of manmade disturbances of a variety of origins (physical: Bouhalloufa and Mexa dams for the Kebir-East and Bouhamdane dam for the Seybouse; chemical: presence of non-point pollutions, and municipal and industrial wastewater).

The climate is typically Mediterranean with a hot and dry summer from June to September, and a cold and rainy winter from October to May.

The substratum of the Kebir-East wadi is composed either of ancient sediments (marls and sandstone) of the Algerian local marine Miocene (equivalent to the continental Aquitanien), degraded slightly on the surface in the east, or more recent Plio-Quaternary sediments corresponding to alluviums of the high and middle terraces of the Kebir-East wadi valley. The recent Quaternary sediments in the valley of KebirEast wadi comprise silt, sand and stones (Marre, 1987).

The watershed of the Seybouse wadi drains water very slowly over a gentle relief from its source in the highlands of Sellawa and Heracta. In the uplands, it flows through a very fractured and complex structured topography, where the hydrographic net is rarely adapted to the structure (Ghachi, 1986). The effluents are torrential and the longitudinal contours are irregular and stretched. The Seybouse River flows through some depressions containing an alluvial water table (C.G.G., 1971; Djabri et al., 2003). This allows regulation of winter precipitations received by the mountain range. When the river reaches the plain of Annaba, it loses its energy and leaves behind a great load of sediments. The geomorphological characteristics of the plain, gentle slope, sand dune barrier, and inundation-prone areas allow the river to flow easily into the Mediterranean Sea.

All chironomid samples were collected with a Surber net $(300 \mathrm{~m}$ mesh size, $50 \mathrm{~cm}$ width). Sampling was carried out during spring (March-May) and summer (July-September) from 2008 to 2011. Ten hauls were made in the opposite sense of the current along the sam-

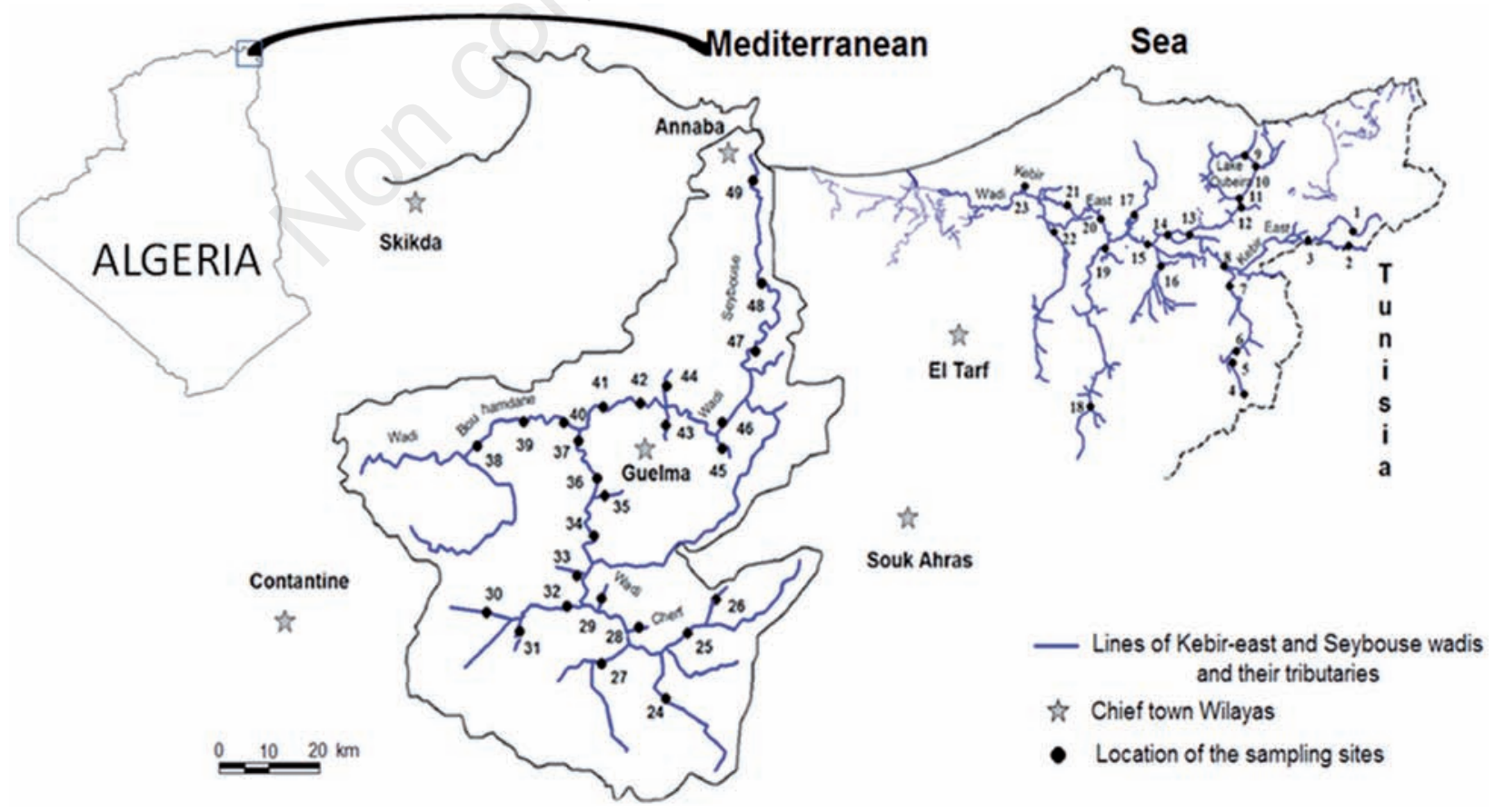

Figure 1. Location of the 49 sampling sites along the Kebir-East and the Seybouse wadis and their tributaries (northeastern Algeria). 
Table 1. List of the sampled sites located along the Kebir-East and Seybouse wadis and their tributaries.

\begin{tabular}{|c|c|c|c|c|c|c|c|c|}
\hline No. & Watercourse & Names of the sampled sites & Code & $\begin{array}{l}\text { Latitude } \\
\text { (N) }\end{array}$ & $\begin{array}{l}\text { Longitude } \\
\text { (E) }\end{array}$ & $\begin{array}{l}\text { Altitude } \\
\text { (m) }\end{array}$ & Substrate size & $\begin{array}{l}\text { Substrate } \\
\text { size classes }\end{array}$ \\
\hline 1 & & O. Leben & LEB & 830'32" & $36^{\circ} 46^{\prime} 56^{\prime \prime}$ & 77 & Very coarse gravel & 5 \\
\hline 2 & & O. Mellili & MEL & 830'28" & $36^{\circ} 46^{\prime} 50^{\prime \prime}$ & 80 & Very fine gravel & 4 \\
\hline 3 & & O. Kebir at R'Mel Souk & RSK & $8^{\circ} 30^{\prime} 10^{\prime \prime}$ & $36^{\circ} 46^{\prime} 55^{\prime \prime}$ & 80 & Very fine gravel & 4 \\
\hline 4 & & O. Louar Amont & LAM & 8०22’58" & $36^{\circ} 36^{\prime} 52^{\prime \prime}$ & 652 & Stones & 6 \\
\hline 5 & & O. Louar Aval & LAV & $8^{\circ} 21^{\prime} 56^{\prime \prime}$ & $36^{\circ} 39^{\prime} 01^{\prime \prime}$ & 200 & Silt & 1 \\
\hline 6 & & O. Bougous Amont & BAM & 8०21'53" & $36^{\circ} 39^{\prime} 06^{\prime \prime}$ & 203 & Very fine gravel & 4 \\
\hline 7 & & O. Bougous Aval & BAV & $8^{\circ} 24^{\prime 2} 27^{\prime \prime}$ & $36^{\circ} 42^{\prime} 36^{\prime \prime}$ & 69 & Very coarse gravel & 5 \\
\hline 8 & & O. Kebir at Ain Assel & KAS & $8^{\circ} 21^{\prime} 57^{\prime \prime}$ & $36^{\circ} 45^{\prime} 59^{\prime \prime}$ & 30 & Very fine gravel & 4 \\
\hline 9 & & Oubéira 3 & OB3 & $8^{\circ} 23^{\prime} 10^{\prime \prime}$ & $36^{\circ} 51^{\prime} 47^{\prime \prime}$ & 24 & Silt & 1 \\
\hline 10 & & Oubéïra 2 & OB2 & $8^{\circ} 25^{\prime} 15^{\prime \prime}$ & $36^{\circ} 51^{\prime} 29^{\prime \prime}$ & 24 & Silt & 1 \\
\hline 11 & & Oubéîra 1 & OB1 & $8^{\circ} 24^{\prime} 12^{\prime \prime}$ & $36^{\circ} 49^{\prime} 29^{\prime \prime}$ & 22 & Silt & 1 \\
\hline 12 & & O. Messida Aval & MAM & $8^{\circ} 24^{\prime} 09^{\prime \prime}$ & $36^{\circ} 49^{\prime} 23^{\prime \prime}$ & 22 & Very fine sand & 2 \\
\hline \multirow[t]{2}{*}{13} & & O. Messida Amont & MAV & $8^{\circ} 22^{\prime} 30^{\prime \prime}$ & $36^{\circ} 47^{\prime} 37^{\prime \prime}$ & 25 & Very fine sand & 2 \\
\hline & Kebir-east wadi & & & & & & & \\
\hline 14 & & O. Kebir Ain Khiar & KAK & $8^{\circ} 18^{\prime} 51^{\prime \prime}$ & $36^{\circ} 46^{\prime} 49^{\prime \prime}$ & 23 & Very coarse sand & 3 \\
\hline 15 & & O. Guergour & GRG & $8^{\circ} 16^{\prime} 52^{\prime \prime}$ & $36^{\circ} 46^{\prime} 32^{\prime \prime}$ & 25 & Very coarse sand & 3 \\
\hline 16 & & O. Kebir Guergour & KGR & $8^{\circ} 16^{\prime} 43^{\prime \prime}$ & $36^{\circ} 46^{\prime} 36^{\prime \prime}$ & 25 & Silt & 1 \\
\hline 17 & & O. Bourdim & BRD & $8^{\circ} 14^{\prime} 50^{\prime \prime}$ & $36^{\circ} 47^{\prime} 22^{\prime \prime}$ & 20 & Very fine gravel & 4 \\
\hline 18 & & O. Zitoun & ZIT & $8^{\circ} 13^{\prime} 02^{\prime \prime}$ & $36^{\circ} 39^{\prime} 06^{\prime \prime}$ & 193 & Stones & 6 \\
\hline 19 & & O. Dardan & DRD & $8^{\circ} 13^{\prime} 16^{\prime \prime}$ & $36^{\circ} 46^{\prime} 39^{\prime \prime}$ & 15 & Silt & 1 \\
\hline 20 & & O. Kebir at Anenes & KAN & $8^{\circ} 12^{\prime} 48^{\prime \prime}$ & $36^{\circ} 47^{\prime} 48^{\prime \prime}$ & 14 & Very fine sand & 2 \\
\hline 21 & & O. Kebir at Righia & KRG & $8^{\circ} 09^{\prime} 35^{\prime \prime}$ & $36^{\circ} 48^{\prime} 51^{\prime \prime}$ & 11 & Silt & 1 \\
\hline 22 & & 0. Boulathan & BLT & $8^{\circ} 06^{\prime} 06^{\prime \prime}$ & $36^{\circ} 49^{\prime} 42^{\prime \prime}$ & 8 & Very fine sand & 2 \\
\hline 23 & & O. Kebir at Sebaa & KSB & $8^{\circ} 09^{\prime} 07^{\prime \prime}$ & $36^{\circ} 48^{\prime} 59^{\prime \prime}$ & 10 & Silt & 1 \\
\hline 24 & & Barrage Sedrata & BSD & $36^{\circ} 03.516^{\prime}$ & $7^{\circ} 27.209^{\prime}$ & 744 & Silt & 1 \\
\hline 25 & & Cherf à Sedrata & CPS & $36^{\circ} 04.479^{\prime}$ & $7^{\circ} 29.640^{\prime}$ & 747 & Silt & 1 \\
\hline 26 & & Oued Krab & OKR & $36^{\circ} 07.210^{\prime}$ & $7^{\circ} 32.780^{\prime}$ & 778 & Silt & 1 \\
\hline \multirow[t]{2}{*}{27} & & Cherf à Ksar Sbahi & CKS & $36^{\circ} 03.207^{\prime}$ & $7^{\circ} 19.557^{\prime}$ & 751 & Silt & 1 \\
\hline & Seybouse wadi & & & & & & & \\
\hline 28 & & Oued El Nil & ONL & $36^{\circ} 08.380^{\prime}$ & $7^{\circ} 26.7311^{\prime}$ & 775 & Very coarse gravel & 5 \\
\hline 29 & & Oued Dbabcha & ODB & $36^{\circ} 12.945^{\prime}$ & $7^{\circ} 19.047^{\prime}$ & 609 & Very coarse gravel & 5 \\
\hline 30 & & Oued el Maleh & OML & $36^{\circ} 08.893^{\prime}$ & $7^{\circ} 8.642$ & 742 & Very fine sand & 2 \\
\hline 31 & & Oued Beni Mheni & OBM & $36^{\circ} 09.207^{\prime}$ & $7^{\circ} 19.557^{\prime}$ & 668 & Very fine sand & 2 \\
\hline 32 & & Barrage Ain Makhlouf & BMK & $36^{\circ} 13.528^{\prime}$ & $7^{\circ} 17.783$ & 643 & Stones & 6 \\
\hline 33 & & Oued El Aare & OAR & $36^{\circ} 13.572^{\prime}$ & $7^{\circ} 19.186^{\prime}$ & 609 & Stones & 6 \\
\hline 34 & & Cherf à Ain Makhlouf & CMK & $36^{\circ} 14.462^{\prime}$ & $7^{\circ} 18.626^{\prime}$ & 600 & Stones & 6 \\
\hline 35 & & Oued Cheniour- Affluent & $\mathrm{OCH}$ & $36^{\circ} 14.877^{\prime}$ & $7^{\circ} 20.610^{\prime}$ & 742 & Stones & 6 \\
\hline 36 & & Cherf à Ain Hsainia & CHS & $36^{\circ} 25.415^{\prime}$ & $7^{\circ} 18.788^{\prime}$ & 270 & Very fine gravel & 4 \\
\hline 37 & & Cherf à Medjez Amar & CMA & $36^{\circ} 26.526^{\prime}$ & $7^{\circ} 18.677^{\prime}$ & 273 & Very fine gravel & 4 \\
\hline 38 & & Bouhamdane à Hammam Debagh & BHD & $36^{\circ} 28.012^{\prime}$ & $7^{\circ} 15.673^{\prime}$ & 305 & Very fine gravel & 4 \\
\hline 39 & & Bouhamdane à Mermoura & BMR & $36^{\circ} 26.522^{\prime}$ & $7^{\circ} 16.292^{\prime}$ & 480 & Stones & 6 \\
\hline 40 & & Bouhamdane à Medjez Amar & BMA & $36^{\circ} 36.592^{\prime}$ & $7^{\circ} 18.615^{\prime}$ & 274 & Very fine gravel & 4 \\
\hline 41 & & Seybouse à Salah SalahSalah & SSS & $36^{\circ} 27.697^{\prime}$ & $7^{\circ} 20.382 '$ & 251 & Stones & 6 \\
\hline 42 & & Seybouse à El-Fedjouj & SFJ & $36^{\circ} 28.893^{\prime}$ & $7^{\circ} 24.926^{\prime}$ & 222 & Stones & 6 \\
\hline 43 & & Oued Zimba - effluent & OZM & $36^{\circ} 26.020^{\prime}$ & $7^{\circ} 18.452^{\prime}$ & 291 & Very fine sand & 2 \\
\hline 44 & & Oued Bradâa & OBR & $36^{\circ} 30.803^{\prime}$ & $7^{\circ} 27.037^{\prime}$ & 285 & Very coarse sand & 3 \\
\hline 45 & & Oued Helia - effluent & $\mathrm{OHL}$ & $36^{\circ} 25.415^{\prime}$ & $7^{\circ} 18.788^{\prime}$ & 144 & Stones & 6 \\
\hline 46 & & Seybouse à Zemzouma & SZM & $36^{\circ} 24.795^{\prime}$ & $7^{\circ} 36.676^{\prime}$ & 143 & Very fine sand & 2 \\
\hline 47 & & Seybouse à Boudaroua & SBD & $36^{\circ} 31.667^{\prime}$ & $7^{\circ} 42.307^{\prime}$ & 100 & Very fine gravel & 4 \\
\hline 48 & & Seybouse à Chihani & SCH & $36^{\circ} 41.002^{\prime}$ & $7^{\circ} 45.527^{\prime}$ & 12 & Very coarse sand & 3 \\
\hline 49 & & Seybouse à Dreân & SDR & $36^{\circ} 39.216^{\prime}$ & $7^{\circ} 46.968^{\prime}$ & 18 & Very fine gravel & 4 \\
\hline
\end{tabular}


Table 2. List of species recorded in 49 sites from two northeastern Algerian watercourses (Kebir-East and Seybouse wadis) between 2007 and 2011. Subfamilies are presented in phylogenetic order and genera in alphabetic order.

Taxa

Frequency of occurrence (\%)

Total abundance of species

\section{Tanypodinae}

103

66

17
Sympotthastia spinifera (Serra-Tosio, 1968) ${ }^{\#}$
Conchapelopia pallidula (Meigen, 1818)*

Procladius choreus (Meigen, 1804) ${ }^{\circ}$

Rheopelopia ornata (Meigen, 1838)\#

Tanypus punctipennis Meigen, $1818^{\circ}$

Zavrelimyia punctatissima (Goetghebuer, 1934)*

Diamesinae

\section{Prodiamesinae}

Prodiamesa olivacea (Meigen, 1818) ${ }^{\circ}$

1.17

0.75
1.12

1.36

0.19

0.01

0.05

Cardiocladius fuscus Kieffer, 1924\#

Corynoneura scutellata Winnertz, $1846^{\circ}$

Cricotopus (Cricotopus) bicinctus (Meigen, 1818) ${ }^{\circ}$

Cricotopus (Isocladius) sylvestris (Fabricius, 1974) ${ }^{\circ}$

Eukiefferiella bedmari Vilchez-Quero \& Laville, 1987*

Eukiefferiella claripennis (Lundbeck, 1890) ${ }^{\circ}$

Eukiefferiella gracei (Edwards, 1929)*

Eukiefferiella ilkleyensis (Edwards, 1929)*

Eukiefferiella sp.1 (Thienemann A., 1926)*

Hydrobaenus distylus (Potthast, 1914)

Hydrobaenus sp.1 Fries, 1830*

Limnophyes minimus (Meigen, 1818)*

Metriocnemus sp.1 Van Der Wulp, 1874*

Orthocladius (Euorthocladius) ashei Soponis, A., 1990*

Orthocladius (Euorthocladius) rivicola Kieffer, 1911

Orthocladius (Orthocladius) excavatus Brundin L., 1947*

Orthocladius (Orthocladius) rubicundus (Meigen, 1818)*

Orthocladius pedestris Kieffer, $1909^{\sharp}$

Paracladius conversus (Walker, 1856)

Parakiefferiella gracillima (Kieffer, 1924)\#

Parametriocnemus stylatus (Kieffer, 1924) ${ }^{\circ}$

Paraphaenocladius sp.1 Thienemann, 1924*

Paratrichocladius rufiventris (Meigen, 1830)*

Paratrissocladius excerptus Walker, $1846^{\#}$

Psectrocladius (Psectrocladius) psilopterus (Kieffer, 1906)*

Psectrocladius sordidellus (Zetterstedt, 1838)

Rheocricotopus chalybeatus (Edwards, 1929)\#

Rheocricotopus fuscipes (Kieffer, 1909)*

Thienemanniella vittata (Edwards, 1924) ${ }^{\circ}$

\section{Tanytarsini}

Cladotanytarsus mancus (Walker, 1856)

Cladotanytarsus sp.1 Kieffer, 1921*

Micropsectra atrofasciata (Kieffer, 1911)\#

Paratanytarsus sp.1 (Thienemann A. \& Bause, 1913)*

Rheotanytarsus sp.1 Thienemann A. \& Bause, 1913*

Tanytarsus sp.1 Van der Wulp, $1874^{\circ}$

\section{Chironominae}

Chironomus plumosus (Linnæus, 1758) ${ }^{\circ}$

Chironomus riparius Meigen, $1804^{\sharp}$

Chironomus sp.1 Meigen, 1803*

Cryptochironomus rostratus Kieffer, $1921^{\#}$

Cryptotendipes sp.1 Beck \& Beck, 1969*

Dicrotendipes nervosus (Stäger, 1839) ${ }^{\circ}$

Einfeldia sp.1 Kieffer, 1924*

Genus near Tribelos*

Glyptotendipes pallens (Meigen, 1804) $)^{\#}$

Harnischia fuscimana (Kieffer, 1921) ${ }^{\circ}$

Microchironomus tener (Kieffer, 1818) \#

Microtendipes pedellus (De Geer, 1776) ${ }^{\circ}$

Paracladopelma camptolabis (Kieffer, 1913)\#

Phaenopsectra flavipes (Meigen, 1818)*

Polypedilum (Tripodura) scalaenum (Schrank, 1803) ${ }^{\circ}$

Polypedilum cultellatum (Goetghebuer, 1931) ${ }^{\circ}$

Polypedilum laetum (Meigen, 1818)*

Polypedilum nubifer (Skuse, 1889)*

Robackia sp.1 Sæther O.A., 1977*

Synendotendipes dispar (Meigen, 1830) \#

Synendotendipes impar (Walker, 1856)*
0.82

0.14

29.52

9.39

0.25

0.8

0.01

0.09

0.02

0.93

0.02

0.01

0.03

0.25

3.15

1.36

0.61

3.18

0.01

0.05

0.27

0.01

2.38

0.12

0.11

0.02

3.32

1.6

0.33

\subsection{8}

0.35

0.06

1.22

0.71

0.51

1.11

2.41

7.15

2.7

0.32

0.11

0.01

0.82

0.01

0.23

0.08

0.1

0.01

0.74

0.01

0.02

3.09

8.7

0.03

4.73

0.02

0.19

0.01
Rheotanytarsus photophilus (Goetghebuer, 1921)

Cryptochironomus defectus (Kieffer, 1913)*

1

4

72

12

2606

829

71

1

8

2

82

2

1

3

22

278

54

281

1

24

1

210

11

10

2

293

29

95

31

5

108

63

45

98

*Species recorded in the Seybouse wadi. ${ }^{\circ}$ Species recorded in both the Kebir-East and the Seybouse wadis. "Species recorded in the Kebir-East. Total abundance was calculated as the abundance from all samples $(\mathrm{n}=49)$ pooled together. 
pling station, in the middle of the current and near the banks. Samples were preserved in 5\% formaldehyde (larvae, pupae), and then examined under a dissecting microscope. The specimens were grouped by morphotypes according to external characteristics visible through the stereomicroscope in the laboratory. Subsequently permanent mounts were prepared in Faure or in Balsam mounting medium, to enable the taxonomic determination of the different morphotypes.

\section{Dataset}

A list of species derived from different studies carried out in northeastern Algeria between 2007 and 2011 (Chaib et al., 2011b) were collected in a database.

The list was based on larval collections, with species identification aided by collection of prepupae and of mature pupae. Italian Keys for larvae determination were used (Ferrarese, 1983; Ferrarese \& Rossaro, 1981; Nocentini, 1985; Rossaro, 1982) along with keys for Palaearctic pupae (Langton \& Visser, 2003).

Substrate size was ranged into six classes (IS0 14688-1) according to the particle size: i) class 1: silt, $<0.063 \mathrm{~mm}$; ii) class 2 : fine sand, 0.063-0.200 mm; iii) class 3: medium-coarse sand, 0.200-2 mm; iv) class 4: fine-medium gravel, 2-20 mm; v) class 5: coarse gravel, 20-63 $\mathrm{mm}$; vi) class 6: cobble, $63-200 \mathrm{~mm}$.

\section{Data analysis}

Quantitative samples collected in the 49 sampling sites were considered in the statistical analyses; the mean abundance of each taxon per site was considered. Rare species were included in the analysis (Smith et al., 2001) resulting in a total of 65 taxa.

Relative abundances of species were transformed by $\log (10)$ to normalize counts. To avoid a problem of logarithm zeroes, the value 1 was added to each abundance. Groups of samples sharing the same type of community composition were defined using a hierarchical cluster analysis (Goodall, 1973) with Ward's linkage method and Euclidian distance measure.

Multi-response permutation procedures (MRPP) (Biondini et al., 1985) were used to test the reliability of the groups obtained.

The species characterizing each cluster were identified with indicator species analysis (IndVal; Dufrêne \& Legendre, 1997). A second Indval analysis was carried out considering the sampling sites grouped based on substrate size. This method combines information on the concentration of species abundances in a particular group and the faithfulness of occurrence of a species in a particular group. Indicator values were tested for statistical significance using a randomization (Monte Carlo) technique (McCune \& Grace, 2002). The significance was tested by carrying out 10,000 Indval analyses.

Agglomerative cluster analysis, MRPP and Indval analysis were performed with the R environment 2.15.1 (R Development Core Team, 2009).

Species richness, diversity and evenness indices were also calculated (Shannon \& Weaver, 1949).

\section{Results}

Sixty-five Chironomidae taxa belonging to four subfamilies were identified (Table 2). Orthocladiinae showed the highest generic richness (29 taxa). This subfamily showed a proportional abundance of $59 \%$, Chironominae $31 \%$. Tanytarsini and Tanypodinae were the less frequent and abundant with approximately $6 \%$ and $5 \%$, respectively.

C. bicinctus showed the highest abundance $\left(2606\right.$ ind $^{*} \mathrm{~m}^{-2}$ ) and frequency of occurrence (29.52\%) and was widespread in almost all the sampling sites (Table 2), followed by $C$. sylvestris and Polypedilum cultellatum with total abundance of 829 and 768, and a frequency of occurrence of $9.39 \%$ and $8.7 \%$, respectively. Total abundance and the fre-

\section{Cluster Dendrogram}

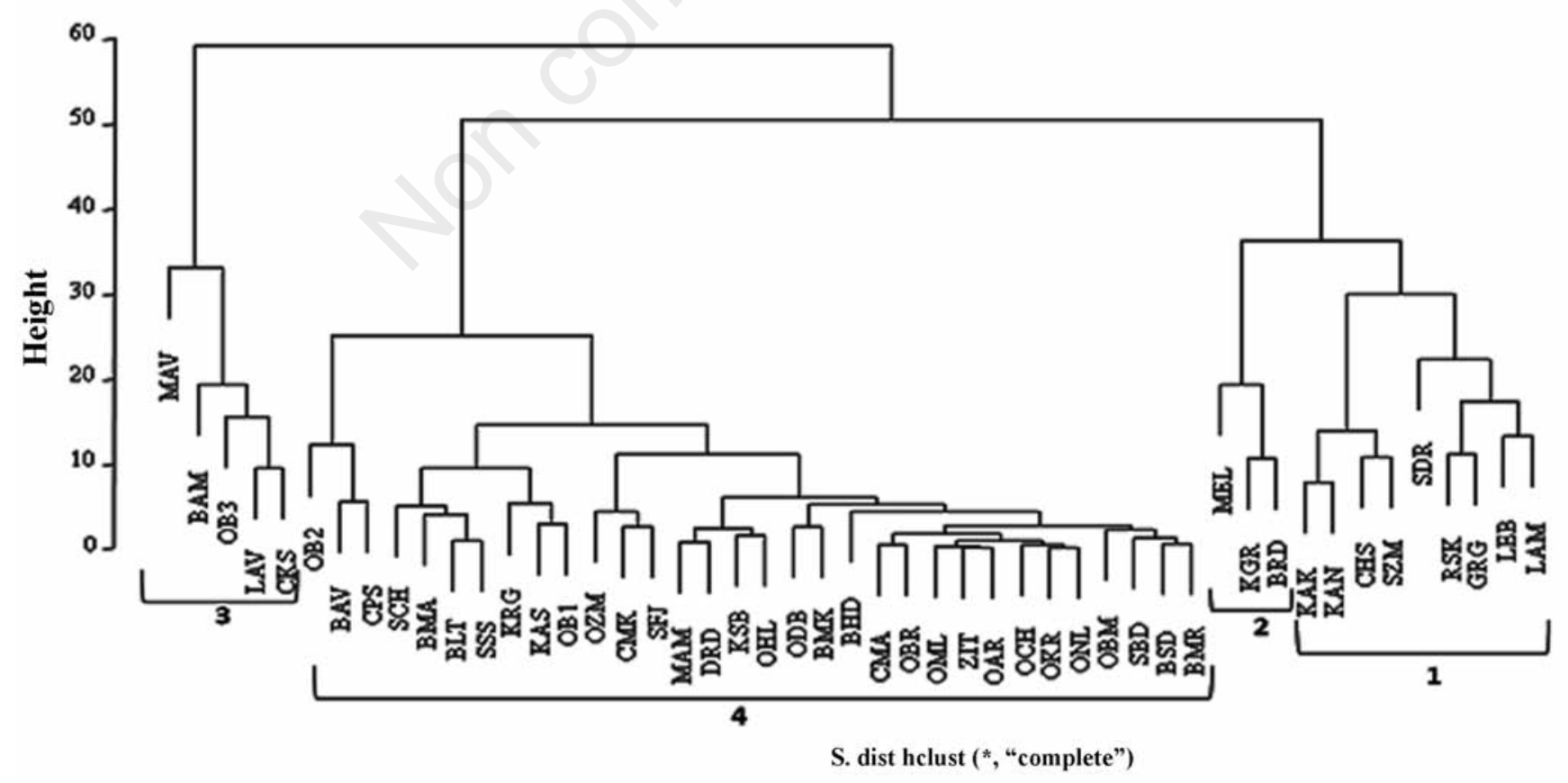

Figure 2. Agglomerative cluster dendrogram based on chironomid communities sampled in the Kebir-East and the Seybouse wadis (2008-2011) (see Table 1 for site codes and names of sampling sites for each group). 
Table 3. Chironomids diversity, richness and evenness for the each sampling sites (see Table 1 for site codes; see Figure 2 for clusters).

\begin{tabular}{|c|c|c|c|c|c|}
\hline No. & Sampled sites & Cluster & Diversity & $\begin{array}{l}\text { Species } \\
\text { richness }\end{array}$ & Evenness \\
\hline 1 & LEB & 1 & 0.75 & 14 & 0.66 \\
\hline 2 & MEL & 2 & 0.64 & 14 & 0.58 \\
\hline 3 & RSK & 1 & 0.81 & 11 & 0.75 \\
\hline 4 & LAM & 1 & 0.75 & 15 & 0.66 \\
\hline 5 & LAV & 3 & 0.81 & 12 & 0.76 \\
\hline 6 & BAM & 3 & 0.63 & 11 & 0.57 \\
\hline 7 & BAV & 4 & 0.80 & 14 & 0.73 \\
\hline 8 & KAS & 4 & 0.79 & 22 & 0.72 \\
\hline 9 & OB3 & 3 & 0.75 & 8 & 0.76 \\
\hline 10 & OB2 & 4 & 0.64 & 9 & 0.64 \\
\hline 11 & $\mathrm{OB} 1$ & 4 & 0.72 & 9 & 0.71 \\
\hline 12 & MAM & 4 & 0.84 & 16 & 0.76 \\
\hline 13 & MAV & 3 & 0.74 & 15 & 0.64 \\
\hline 14 & KAK & 1 & 0.64 & 11 & 0.54 \\
\hline 15 & GRG & 1 & 0.62 & 11 & 0.59 \\
\hline 16 & KGR & 2 & 0.62 & 12 & 0.54 \\
\hline 17 & BRD & 2 & 0.80 & 14 & 0.72 \\
\hline 18 & ZIT & 4 & 0.15 & 5 & 0.23 \\
\hline 19 & DRD & 4 & 0.82 & 13 & 0.76 \\
\hline 20 & KAN & 1 & 0.75 & 9 & 0.73 \\
\hline 21 & KRG & 4 & 0.71 & 12 & 0.59 \\
\hline 22 & BLT & 4 & 0.73 & 11 & 0.66 \\
\hline 23 & KSB & 4 & 0.76 & 12 & 0.70 \\
\hline 24 & BSD & 4 & 0.25 & 9 & 0.28 \\
\hline 25 & CPS & 4 & 0.83 & 19 & 0.72 \\
\hline 26 & OKR & 4 & 0.77 & 9 & 0.77 \\
\hline 27 & CKS & 3 & 0.90 & 18 & 0.86 \\
\hline 28 & ONL & 4 & 0.78 & 8 & 0.85 \\
\hline 29 & ODB & 4 & 0.89 & 15 & 0.87 \\
\hline 30 & OML & 4 & 0.83 & 9 & 0.89 \\
\hline 31 & OBM & 4 & 0.63 & 9 & 0.61 \\
\hline 32 & BMK & 4 & 0.72 & 7 & 0.77 \\
\hline 33 & OAR & 4 & 0.75 & 4 & 1.00 \\
\hline 34 & CMK & 4 & 0.89 & 20 & 0.83 \\
\hline 35 & $\mathrm{OCH}$ & 4 & 0.77 & 23 & 0.68 \\
\hline 36 & CHS & 1 & 0.81 & 17 & 0.74 \\
\hline 37 & CMA & 4 & 0.89 & 20 & 0.85 \\
\hline 38 & BHD & 4 & 0.73 & 10 & 0.75 \\
\hline 39 & BMR & 4 & 0.80 & 11 & 0.81 \\
\hline 40 & BMA & 4 & 0.70 & 17 & 0.64 \\
\hline 41 & SSS & 4 & 0.85 & 17 & 0.78 \\
\hline 42 & SFJ & 4 & 0.89 & 17 & 0.86 \\
\hline 43 & OZM & 4 & 0.84 & 23 & 0.72 \\
\hline 44 & OBR & 4 & 0.77 & 12 & 0.76 \\
\hline 45 & OHL & 4 & 0.78 & 19 & 0,74 \\
\hline 46 & SZM & 1 & 0.85 & 17 & 0.78 \\
\hline 47 & SBD & 4 & 0.76 & 15 & 0.74 \\
\hline 48 & SCH & 4 & 0.81 & 16 & 0.78 \\
\hline 49 & SDR & 1 & 0.81 & 15 & 0.73 \\
\hline
\end{tabular}

Table 4. Chironomid indicator species by group of sites and substrate size (Indval analysis).

$\begin{array}{cc}\text { Taxa } & \text { Substrate } \\ \text { assemblages } & \text { sive } \\ \text { Taxa } & \text { Max P Max P } \\ \text { class } & \text { class }\end{array}$

\section{Tanypodinae}

Conchapelopia pallidula

Procladius choreus

Rheopelopia ornata

Tanypus punctipennis

Zavrelimyia punctatissima

$\begin{array}{llll}4 & 0.378 & 6 & 0.324 \\ 3 & 0.102 & 1 & 0.235 \\ 3 & 0.144 & 5 & 0.575 \\ 1 & 0.749 & 4 & 0.519 \\ 4 & 0.292 & 3 & 0.581\end{array}$

Diamesinae
Sympotthastia spinifera

Prodiamesinae

Prodiamesa olivacea

\section{Orthocladiinae}

Cardiocladius fuscus

Corynoneura scutellata

Cricotopus (Cricotopus) bicinctus

Cricotopus (Isocladius) sylvestris

Eukiefferiella bedmari

Eukiefferiella claripennis

Eukiefferiella gracei

Eukiefferiella ilkleyensis

Eukiefferiella sp.1

Hydrobaenus distylus

Hydrobaenus sp.1

Limnophyes minimus

Metriocnemus sp.1

Orthocladius (Euorthocladius) ashei

Orthocladius (Euorthocladius) rivicola

Orthocladius (Orthocladius) excavatus

Orthocladius (Orthocladius) rubicundus

Orthocladius pedestris

Paracladius conversus

Parakiefferiella gracillima

Parametriocnemus stylatus

Paraphaenocladius sp.1*

Paratrichocladius rufiventris

Paratrissocladius excerptus

Psectrocladius (Psectrocladius) psilopterus

Psectrocladius sordidellus

Rheocricotopus chalybeatus

Rheocricotopus fuscipes

Thienemanniella vittata

\section{Tanytarsini}

Cladotanytarsus mancus

Cladotanytarsus sp.1

Micropsectra atrofasciata

Paratanytarsus sp.1

Rheotanytarsus photophilus

Rheotanytarsus sp.1

Tanytarsus sp.1

\begin{tabular}{llll}
2 & 0.057 & 4 & 0.746 \\
\hline
\end{tabular}

Chironominae

Chironomus plumosus

Chironomus riparius

Chironomus sp.1

Cryptochironomus defectus

Cryptochironomus rostratus

Cryptotendipes sp.1

Dicrotendipes nervosus

Einfeldia sp.1

Genus near Tribelos

Glyptotendipes pallens

Harnischia fuscimana

Microchironomus tener

Microtendipes pedellus

Paracladopelma camptolabis

Phaenopsectra flavipes

Polypedilum (Tripodura) scalaenum

Polypedilum cultellatum

Polypedilum laetum

Polypedilum nubifer

Robackia sp.1

Synendotendipes dispar

\begin{tabular}{ccccc} 
Synendotendipes impar & 4 & 1 & 4 & 0.747 \\
\hline Significant values $(\mathrm{P}<0.05)$ are in italics (see Figure 2 for groups of sites: cluster analysis). ${ }^{*}$ Species
\end{tabular}

$\begin{array}{llll}4 & 0.719 & 4 & 0.356\end{array}$

$\begin{array}{llll}2 & 0.042 & 3 & 0.417\end{array}$

$\begin{array}{llll}2 & 0.042 & 3 & 0.417 \\ 2 & 0.643 & 4 & 0.032 \\ 2 & 0.011 & 4 & 0.510\end{array}$

$\begin{array}{llll}1 & 0.029 & 5 & 0.809\end{array}$

$\begin{array}{llll}4 & 0.029 & 5 & 0.809 \\ 2 & 0.6912 & 0.571\end{array}$

$\begin{array}{llll}2 & 0.112 & 6 & 0.923\end{array}$

$\begin{array}{llll}4 & 1.000 & 4 & 0.776 \\ 4 & 1.000 & 6 & 0.270\end{array}$

$\begin{array}{llll}4 & 1.000 & 6 & 0.270 \\ 4 & 1.000 & 6 & 0.898\end{array}$

$\begin{array}{llll}2 & 0.116 & 4 & 0.807\end{array}$

$\begin{array}{llll}4 & 1.000 & 3 & 0.455\end{array}$

$\begin{array}{llll}3 & 0.164 & 1 & 1.000 \\ 3 & 0.390 & 1 & 0.757\end{array}$

$\begin{array}{llll}3 & 0.537 & 6 & 0.745\end{array}$

$\begin{array}{llll}3 & 0.534 & 6 & 0.745 \\ 4 & 0.609 & 2 & 0.796 \\ 3 & 0.750 & 6 & 0.342\end{array}$

$\begin{array}{llll}3 & 0.750 & 6 & 0.502\end{array}$

$\begin{array}{llll}3 & 0.750 & 6 & 0.502 \\ 2 & 0.007 & 4 & 0.129\end{array}$

$\begin{array}{llll}4 & 1.000 & 2 & 0.314\end{array}$

$\begin{array}{llll}2 & 0.114 & 4 & 0.271\end{array}$

$\begin{array}{llll}2 & 0.572 & 4 & 0.297\end{array}$

$\begin{array}{llll}4 & 1.000 & 6 & 0.538\end{array}$

$\begin{array}{llll}4 & 0.265 & 3 & 0.542\end{array}$

$\begin{array}{llll}3 & 0.941 & 6 & 0.057\end{array}$

$\begin{array}{llll}4 & 0.724 & 2 & 0.497 \\ 1 & 0.569 & 2 & 0.725\end{array}$

$\begin{array}{llll}1 & 0.569 & 2 & 0.725 \\ 2 & 0.009 & 3 & 0.971\end{array}$

$\begin{array}{llll}2 & 0.009 & 3 & 0.971 \\ 4 & 0.387 & 6 & 0.118\end{array}$

$\begin{array}{llll}4 & 0.387 & 6 & 0.118 \\ 2 & 0.533 & 1 & 0.139\end{array}$ 
quency of occurrence were calculated from all samples $(n=49)$ pooled together.

Species Richness ranged from 4 (site 33 ) to 23 (sites 35 and 43), diversity between 0.15 (site 18) and 0.90 (site 27). Evenness values ranged from 0.23 (site 18) to 1 (site 33 ) (Table 3 ).

Indicator species were determined for each group of sites and then according to substrate-type. Indicator values are in Table 4, along with statistical significance values calculated by randomization (Monte Carlo) (McCune \& Grace, 2002).

The agglomerative cluster analysis (Figure 2) grouped the sampling sites into clusters according to the chironomid species. A 4-group level of the dendrogram was chosen, the MRPP results showing that the groups obtained were statistically different $(\mathrm{A}=0.302, \mathrm{P}<0.005)$.

Corynoneura scutellata and Dicrotendipes nervosus showed the lowest $P$ values ( 0.032 and 0.033 , respectively) and seemed to have a preference to very fine gravel, Glyptotendipes pallens presented a P value of 0.036 suggesting a preference for very fine sand substrate (Table 1).

It should be emphasized that the number of larvae recorded of $C$. scutellata and $G$. pallens was very low; only a few larvae were captured in some stations. D. nervosus was more abundant in stations KAS (20 larvae) and SDR (12 larvae).

\section{Discussion and conclusions}

The percentage distribution of taxa within chironomid subfamilies was in accordance with previous studies (Moubayed et al., 2007; Chaib et al., 2011b), with Orthocladiinae as the most frequent, taxon-richest and abundant subfamily.

C. bicinctus was the most abundant and widely distributed taxon, confirming that the species can tolerate a wide range of substrate size (with a preference for the sandy substrate). In contrast $C$. scutellata was the least abundant, present only in very fine gravel substrates.

The cobble and gravel substrates held the highest number of chironomids among all substrates (Table 1). It was similarly observed (Campbell \& Meadows, 1972) that cobble substrates offer the most suitable microenvironments because they supply materials used by the larvae to construct runways and to build cases about their bodies, crevices for protection, sources of attachment, they are also a source of food since rock surfaces are covered with periphyton (mosses and algae).

In contrast, silty stations housed the least number of chironomids (Table 1) due to the fact that they had the least favorable physical conditions, such as high current and low values of organic matter and transparency. However, $P$. cultellatum was found to be dominant there.

\section{References}

ANNANI F., ALFARHAN A.H., SAMRAOUI B., 2012 - Aquatic Hemiptera of northeastern Algeria: distribution, phenology and conservation. - Rev. Ecol. Terre Vie. [In press].

ARAB A., LEK S., LOUNACI A., PARK YS., 2004 - Spatial and temporal patterns of benthic invertebrate communities in an intermittent river (North Africa). - Ann. Limnol. 40: 317-327.

BATTISTI C., LUISELLI L., PANTANO D., TEOFILI C., 2008 - On threats analysis approach applied to a Mediterranean remnant wetland: is the assessment of human-induced threats related to different level of expertise of respondents? - Biodivers. Conserv. 17: 1529-1542.

BELAIDI N., TALEB A., GAGNEUR J., 2004 - Composition and dynamics of hyporheic and surface fauna in a semi-arid stream In relation to the management of a polluted reservoir. - Ann. Limnol. 40: 237-248. BIONDINI M.E., BONHAM C.D., REDENTE E.F., 1985 - Secondary suc- cessional patterns in a sage brush (Artemisia tridentata) community as they relate to soil disturbance and soil biological activity. Vegetation 60: 25-36.

BR00KS A.J., HAEUSLER T., REINFELDS I., WILLIAMS S., 2005 Hydraulic microhabitats and the distribution of macroinvertebrate assemblages in riffles. - Freshwat. Biol. 50: 331-344.

CAMPBELL J.L., MEADOWS P.S., 1972 - An analysis of aggregations formed by the caddis fly larvae. - J. Zool. Lond. 167: 133-134.

C.G.G., 1971 - Prospection géophysique de la plaine alluviale de l'Oued Seybouse et ses affluents: région de Guelma. General Campaign of Geophysics. - Rapport interne.

CHAIB N., SAMRAOUI B., 2011 - Evaluation de la qualité physico-chimique des eaux de l'oued Kébir-Est et de ses principaux affluents (Nord-Est algérien). - Sécheresse 22: 171-177.

CHAIB N., ALFARHAN A.H., AL-RASHEID K.A.S., SAMRAOUI B., 2011a Environmental determinants of diatom assemblages along a North African wadi, the Kebir-East, northeast Algeria. - J. Limnol. 70: 33-40.

CHAIB N., SAMRAOUI B., MARZIALI L., ROSSARO B., 2011b Chironomid taxocenosis in a south Mediterranean wadi, the KebirEast. - Studi Trentini di Scienze Naturali 89: 29-34.

COFFMAN W.P., FERRINGTON JR. L.C., 1984 - Chironomidae. In: MERRITT R.W., CUMMINS K.W., eds., An introduction to the aquatic insects of North America. 2nd ed. - Kendall Hunt Publishing Co., Dubuque: 551-652.

CRANSTON P.S., 1995 - Biogeography. In: ARMITAGE P.D., CRANSTON P.S., PINDER, L.C.V., eds., The Chironomidae: biology and ecology of non-biting midges. - Chapman and Hall, London: 62-84.

DJABRI L., HANI A., LAOUAR R., MANIA J., MUDRY J., LOUHI A., 2003 - Potential pollution of groundwater in the valley of the Seybouse River, north-eastern Algeria. - Environ. Geol. 44: 738-744.

DUFRÊNE M., LEGENDRE P., 1997 - Species assemblages and indicator species: the need for a flexible asymmetrical approach. - Ecol. Monographs 67: 345-366.

GIBBS J.P., 2000 - Wetland loss and biodiversity conservation. Conserv. Biol. 14: 314-317.

GHACHI A., 1986 - Hydrologie et utilisation de la ressource en eau: Bassin de la Seybouse. - Office des publications universitaires (OPU), Alger: 508 pp.

FERRARESE U., 1983 - Chironomidi, 3 (Diptera, Chironomidae: Tanypodinae) 26. In: RUFFO S., ed., Guide per il riconoscimento delle specie animali delle acque interne italiane, CNR/AQ/1/204. Consiglio Nazionale delle Ricerche, Roma: 67.

FERRARESE U., ROSSARO B., 1981 - Chironomidi, 1 (Diptera, Chironomidae: Generalità, Diamesinae, Prodiamesinae) 12. In: RUFFO S., ed., Guide per il riconoscimento delle specie animali delle acque interne italiane, CNR/AQ/1/129. - Consiglio Nazionale delle Ricerche, Roma: 97.

GO0DALL D.W., 1973 - Numerical classification. In: WHITTAKER RH, ed., Handbook of vegetation science, part 5: Ordination and classification of communities. - Dr W Junk, The Hague: 575-615.

HOLLIS G.E., 1992 - The causes of wetland loss and degradation in the Mediterranean. In: FINLAYSON C.M., DAVIS T.J., eds., Managing Mediterranean wetlands and their birds: vol. 20. - IWRB, Slimbridge: 83-90.

HULME M., DOHERTY R.M., NGARA, T., NEW M.G., LISTER D., 2001 African climate change: 1900-2100. - Climate Res. 17: 145-168.

KHELIFA R., YOUCEFI AJ., KAHLERRAS A., AL FARHAN A., AL RASHEID KAS., SAMRAOUI B., 2011- L'odonatofaune (Insecta : Odonata) du bassin de la Seybouse en Algérie : intérêt pour La biodiversité du Maghreb. - Rev. Ecol. TerreVie 66: 55-66.

LANGTON P.H., VISSER H., 2003 - Chironomidae exuviae. A Key to the pupal exuviae of west Palaearctic Region. World biodiversity database CD-ROM series. - Expert Center for Taxonomic Identification, University of Amsterdam. Available from: http://etiis.org.uk/ 
LEGENDRE P., LEGENDRE L., 1998 - Numerical ecology, 2nd English edition. Elsevier Science BV, Amsterdam: 853 pp.

LOUNACI A., BROSSE S., MOULOUD S.A., LOUNACI-DAOUDI D., MEBARKI N., THOMAS A., 2000a - Current knowledge of benthic Macroinvertebrate diversity in Algerian stream, a species check list of the Sébaou river basin (Tizi Ouzou). - Bull. Soc. Hist. Nat. (Toulouse) 136: 43-55.

LOUNACI A., BROSSE S., THOMAS A., LEK S., 2000b - Abundance, diversity and community structure of macroinvertebrates in an Algerian stream: the Sebaou wadi. - Ann. Limnol. 36: 123-133.

MARRE A., 1987 - Le Tell oriental algérien de Collo à la frontière tunisienne: Etude géomorphologique. - Digree Diss., Univ. Aix-en Provence: 463 pp.

MCGEOCH M.A., CHOWN S.L., 1998 - Scaling up the value of bioindicators. - Trend Ecol. Evol. 13: 46-47.

MCCUNE B., GRACE J.B., 2002 - Analysis of ecological communities. Gleneden Beach, Oregon: MjM Software.

MOUBAYED J., LOUNACI A., LOUNACI DAOUDI D., 2007 - Non-biting midges from Algeria, North Africa (Diptera, Chironomidae). Ephemera 8: 93-99.

NOCENTINI A., 1985 - Chironomidi, 4 (Diptera, Chironomidae: Chironominae, larvae) 29. In: RUFFO S., ed., Guide per il riconoscimento delle specie animali delle acque interne italiane, CNR/AQ/1/233. - Consiglio Nazionale delle Ricerche, Roma: 186 pp.

R DEVELOPMENT CORE TEAM, 2009 - R: a language and environment for statistical computing. - R Foundation for Statistical Computing, Vienna.

ROSSARO B., 1982 - Chironomidi, 2 (Diptera, Chironomidae:
Orthocladiinae) 16. In: RUFFO S., ed., Guide per il Riconoscimento delle specie animali delle Acque Interne Italiane, C.N.R./AQ/1/171. - Consiglio Nazionale delle Ricerxche, Roma: 80 pp.

SAMRAOUI B., CORBET P.S., 2000 - The Odonata of Numidia. Part I: status and distribution. - Int. J. Odonatol. 3: 11-25.

SAMRAOUI B., MENAI R., 1999 - A contribution to the study of Algerian Odonata. - Int. J. Odonatol. 2: 145-165.

SAMRAOUI B., SAMRAOUI F., 2008 - An ornithological survey of the wetlands of Algeria: Important Bird Areas, Ramsar sites and threatened species. - Wildfowl 58: 71-98.

SAMRAOUI B., SAMRAOUI F., BENSLIMANE N., ALFARHAN A., ALRASHEID, K.A.S., 2012 - A precipitous decline of the Algerian Newt Pleurodeles poireti Gervais, 1835 and other changes in the status of amphibians of Numidia, North-Eastern Algeria. - Rev. Ecol. Terre Vie 67: 71-82.

SANSEVERINO A.M., NESSIMIAN J.L., 2001 - Habitats de larvas de Chironomidae (Insecta, Diptera) em riachos de Mata Atlântica no Estado do Rio de Janeiro. - Acta Limnol. Bras. 13: 29-38.

SHANNON C.E., WEAVER W., 1949 - The mathematical theory of communication. - University of Illinois Press, Urbana, IL.

SMITH H., WOOD P.J., GUNN J., 2001 - The macroinvertebrate communities of limestone springs in the Wye Valley, Derbyshire Peak District, UK. - Cave Karst Sci. 28: 67-78.

TICKNER D., ARMITAGE P.D., BICKERTON M.A., HALL K.A., 2000 Assessing stream quality using information on mesohabitat distribution and character. - Aquatic Conserv. Mar. Freshw. Ecosyst. 10: $170-196$. 